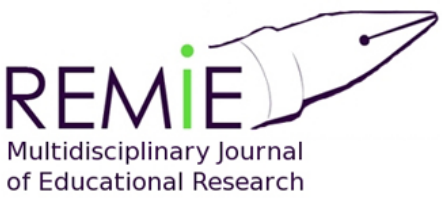

Multidisciplinary Journal

of Educational Research
Hipatia Press

www.hipatiapress.com

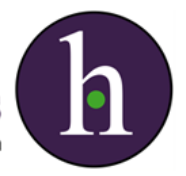

Instructions for authors, subscriptions and further details:

http://remie.hipatiapress.com

\title{
Análisis del Liderazgo Electrónico y la Competencia Digital del Profesorado de Cooperativas Educativas de Andalucía (España)
}

Jesús López Belmonte ${ }^{1}$, Santiago Pozo Sánchez² ${ }^{2}$ Arturo Fuentes Cabrera $^{2}$, José María Romero Rodríguez²

1) Universidad Internacional de Valencia. España

2) Universidad de Granada. España

Date of publication: June $15^{\text {th }}, 2019$

Edition period: February 2019-June 2019

To cite this article: López, J., Pozo, S., Fuentes, A., y Romero, JM. (2019). Análisis del Liderazgo Electrónico y la Competencia Digital del Profesorado de Cooperativas Educativas de Andalucía (España). Multidisciplinary Journal of Educational Research, 9(2), 194-223. doi:

10.4471/remie.2019.4149

To link this article: http://dx.doi.org/10.447/remie.2019.4149

\section{PLEASE SCROLL DOWN FOR ARTICLE}

The terms and conditions of use are related to the Open Journal System and to Creative Commons Attribution License (CC-BY). 


\section{Análisis del Liderazgo Electrónico y la Competencia Digital del Profesorado de Cooperativas Educativas de Andalucía (España)}

Jesús López Belmonte

Universidad Internacional de

Valencia

Arturo Fuentes Cabrera

Universidad de Granada

\author{
Santiago Pozo Sánchez \\ Universidad de Granada
}

José María Romero Rodríguez

Universidad de Granada

\section{Resumen}

La irrupción de las tecnologías de la información y la comunicación (TIC) en la educación es una realidad presente, por lo que resulta fundamental que el profesorado disponga de un nivel adecuado de competencia digital con el propósito de desplegar una praxis innovadora en su labor como docente. Un aspecto relevante es el ejercicio de formación continua en materia tecnológica que efectúe para mejorar sus destrezas digitales. En todo esto juega un papel importante el fenómeno del liderazgo tecnológico que permita una dinamización e influencia de los profesionales hacia la formación, inclusión y aplicación de recursos innovadores en los espacios de aprendizaje. El objetivo de este trabajo fue conocer la incidencia del liderazgo tecnológico de los docentes de las cooperativas de enseñanza de Andalucía (España). Para ello, se aplicó una metodología cuantitativa descriptiva y correlacional, utilizando como instrumento de recogida de datos un cuestionario en una muestra de 428 docentes. Entre los resultados, se encuentran deficiencias formativas en materia TIC, desigualdades competenciales tanto en las áreas de la competencia digital docente como en las cualidades para desplegar un liderazgo tecnológico, lo que desemboca en una escasez de e-líderes. Los docentes revelan que hacen un uso combinado (presencial e innovador) a nivel metodológico. Por último, se hallaron diferencias entre sexos, tanto en las variables tomadas en el liderazgo como en las diversas áreas que articulan la competencia digital.

Palabras Clave: competencias digitales, educación tecnológica, formación de profesores, liderazgo docente, tecnologías de la información y la comunicación. 
No. 2 June 2019, pp. 194-223

\title{
Analysis of electronic leadership and digital competence of teachers of educational cooperatives in Andalucía (Spain)
}

Jesús López Belmonte

Valencian International

University

Arturo Fuentes Cabrera

University of Granada
Santiago Pozo Sánchez

University of Granada

José María Romero Rodríguez

University of Granada

\begin{abstract}
The irruption of information and communication technologies (ICT) in education is a present reality, so it is essential that teachers have an adequate level of digital competence in order to deploy innovative praxis in their work as teacher. A relevant aspect is the exercise of continuous training in technological matters that is carried out to improve their digital skills. In all this plays an important role the phenomenon of technological leadership that allows a dynamic and influence of professionals towards training, inclusion and application of innovative resources in learning spaces. The objective of this work was to know the incidence of the technological leadership of the teachers of the teaching cooperatives of Andalusia (Spain). For this, a descriptive and correlational quantitative methodology was applied, using as a data collection instrument a questionnaire in a sample of 428 teachers. Among the results, training deficiencies found in ICT, competence inequalities both in the areas of teaching digital skills and qualities to deploy technological leadership, which leads to a shortage of e-leaders. The teachers reveal that they make a combined use (face-to-face and innovative) at the methodological level. Finally, differences were found between the sexes, both in the variables taken in the leadership and in the different areas that articulate the digital competence.
\end{abstract}

Keywords: digital skills, technological education, teacher training, teacher leadership, information and communication technologies 


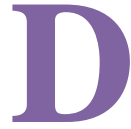

urante los últimos años se han producido una serie de transformaciones cruciales en aspectos tan relevantes como la población, la economía o la tecnología, lo que ha propiciado un cambio altamente significativo en el panorama educativo (Rotellar y Cain, 2016). El acceso a la información, a los contenidos y al conocimiento se produce de una forma muy distinta a la que se realizaba años atrás (López y Bernal, 2019), de manera que se erige como necesaria una transformación en las metodologías pedagógicas para que se adecuen a la realidad actual, una realidad centrada en las tecnologías de la información y la comunicación (TIC), en el aprendizaje virtual y en la digitalización del proceso instructor (Aróstegui y Guerrero, 2014; McCarthy, 2016; Vaillant, Rodríguez y Bernasconi, 2017).

Bajo este panorama social, nos encontramos con una clara tendencia actual encaminada hacia la optimización del proceso de enseñanza y aprendizaje, hecho que se encuadra en una reformulación del rol del discente, que debe cambiar de pasivo a activo para poder alcanzar con éxito los objetivos curriculares (Cerda y Saiz, 2018; Jovanović, Gašević, Dawson, Pardo y Mirriahi, 2017; Sola, Aznar, Romero y Rodríguez-García, 2019), y que - por consiguiente- el proceso de enseñanza resulte eficaz y pragmático para los docentes y los discentes (Martín, García y Muñoz, 2014). De esta forma, se observa durante la última década un importante esfuerzo a nivel estatal para incluir las TIC dentro de los centros educativos (Area, Hernández y Alonso, 2016) y también por parte de los propios centros para incorporar la tecnología dentro de sus proyectos (Fernández, Fernández y Rodríguez, 2018) para trabajar la competencia digital de manera eficaz (Méndez y Delgado, 2016). En definitiva, la educación se dirige hacia un proceso de renovación de sus principios más básicos (González, 2017), una auténtica revolución en la manera de enseñar y aprender, dirigida hacia un uso correcto y responsable de la tecnología para poder hacer realidad la inmersión del discente en el mundo digital (Cabero, 2017; Viñals y Cuenca, 2016).

A pesar de ello, los esfuerzos por parte de las instituciones educativas para incorporarse a las nuevas tendencias digitales del aprendizaje deben venir acompañados de una renovación de la forma en la que se lleva a cabo el proceso instructor, siendo los docentes los principales protagonistas para que dicha incorporación se produzca de forma efectiva. En esta línea, el docente 
debe integrar en su corpus metodológico propuestas pedagógicas en las que el discente adquiera un rol activo y el docente se erija como un mero guía y orientador del aprendizaje (Pozuelo, 2014), que poniendo en práctica su liderazgo electrónico (e-liderazgo) podrá fomentar una actitud positiva hacia la tecnología entre sus compañeros docentes (Macías, Chum, Aray y Rodríguez, 2018) y así pueda finalmente efectuarse una inclusión significativa de las TIC en los espacios de instrucción a partir de las tecnologías emergentes (Aznar, Cáceres y Romero, 2018; Rodríguez y Pedraja, 2017).

En este sentido, nos encontramos con una realidad en la que las TIC se encuentran inmersas cada vez más en el ámbito educativo, permitiendo una serie de mejoras en los procesos de enseñanza y aprendizaje (Viñals y Cuenca, 2016), gracias a la incorporación de dispositivos tecnológicos y de espacios digitales (Kumar y Kumar, 2018; McGill, Koppi y Armarego, 2014; Murillo, Garrido y Hernández, 2016; Vacek y Rybenska, 2015) que permiten fomentar y mejorar el aprendizaje significativo (Maquilón, Mirete y Avilés, 2017) constructivista (Mingorance, Trujillo, Cáceres y Torres, 2017), la motivación y los resultados académicos (Laskaris, Kalogiannakis y Heretakis, 2017).

No obstante, siguiendo a Díaz (2013), la tecnología educativa también supone una serie de desventajas, entre las que destacan: a) la infoxicación supone una labor ardua para el docente al tener que seleccionar y analizar los contenidos expuestos en la red; b) Distracción de los discentes como consecuencia del gran volumen de información de internet; c) La tecnología está alcanzando un alto coste el cual dificulta su inclusión en el plano educativo; d) Supone un ejercicio constante de actualización y protección; e) En determinadas ocasiones presentan problemas que dificultan la labor docente.

A pesar de lo expuesto, es necesario considerar que esta actitud de bonanza hacia la utilización de lo tecnológico requiere fortalecer de forma efectiva la competencia digital docente.

Aproximándonos al concepto de competencia digital, resulta necesario señalar que su origen se imbrica dentro de la denominada Ponencia de la Competencia Digital Docente organizada por el Instituto Nacional de Tecnologías Educativas y de Formación del Profesorado (INTEF, 2017). El 


\section{López, Pozo, Fuentes, \& Romero-Liderazgo Digital}

principal objetivo de dicha ponencia consistía en conformar un Marco Común De Competencia Digital Docente (Blanco, 2018) con base en los principios formulados en el Marco estratégico de Educación y Formación, y en las recomendaciones propuestas por el Parlamento Europeo (Comisión Europea, 2016; Comisión Europea, 2009). De esta forma, se produjo una estandarización que sirvió para que la competencia digital fuese contemplada bajo el mismo criterio y de forma idéntica por parte de todo el colectivo docente. Se produce, así, una estructuración en seis niveles (A1, A2, B1, B2, C1 y C2), 21 competencias y cinco áreas (Durán, Gutiérrez y Prendes, 2016; Esteve, 2016), organizadas tal y como se muestra en la tabla 1.

La literatura científica muestra una evidente abundancia productiva en lo que a investigaciones sobre competencia digital docente se refiere (Falcó, 2017; Fernández y Fernández, 2016; González y Gutiérrez, 2017; Pérez y Rodríguez, 2016; Rodríguez-García, Cáceres y Alonso, 2018). A pesar de ello, resulta necesario el desarrollo de un corpus científico que se adentre en la cuantificación del nivel de los docentes actuales en lo concerniente a competencia digital, para poder conocer aquellas en las que el profesorado muestre un nivel más alto y poder trabajar con aquellas en las que los docentes demuestren un nivel más bajo, teniendo en cuenta que los bajos niveles competenciales no solo dependen de la iniciativa docente o de los planes de formación continua.

Estudios recientes (Fernández, Fernández y Rodríguez, 2018; Fernández y Rodríguez, 2017; Fuentes, López y Pozo, 2019; Valdés y Gutiérrez, 2018) hallan carencias competenciales a nivel digital entre los docentes analizados. Diversas investigaciones han constatado varios factores determinantes que influyen en los niveles de la competencia digital docente: a) la formación inicial de los estudiantes universitarios que se convertirán en futuros docentes (Martínez, 2018; Moreno, Gabarda y Rodríguez, 2018; Peirats, Marín, Granados y Morote, 2018); b) la falta de recursos tecnológicos que sufre un importante número de docentes dentro de los centros educativos (Area y Guarro, 2012; Gallego, Gámiz y Gutiérrez, 2010; Suárez, Almerich, Díaz y Fernández, 2012); c) la formación continua del profesorado en materia TIC, que - a pesar de realizar cursos de dicha índole-, su número no resulta suficiente (Fuentes, López y Pozo, 2019). 
Tabla 1:

Áreas de la competencia digital

\begin{tabular}{|c|c|c|}
\hline & $\begin{array}{l}\text { Área } \\
\text { competencial }\end{array}$ & Competencia digital \\
\hline \multirow[t]{2}{*}{ Área 1} & \multirow{2}{*}{$\begin{array}{l}\text { Información y } \\
\text { alfabetización } \\
\text { informacional }\end{array}$} & $\begin{array}{l}\text { Navegación, búsqueda y filtrado de } \\
\text { información, datos y contenidos digitales } \\
\text { Evaluación de información, datos y } \\
\text { contenidos digitales }\end{array}$ \\
\hline & & $\begin{array}{l}\text { Almacenamiento y recuperación de } \\
\text { información, datos y contenidos digitales }\end{array}$ \\
\hline \multirow[t]{2}{*}{ Área 2} & \multirow[t]{2}{*}{$\begin{array}{l}\text { Comunicación y } \\
\text { colaboración }\end{array}$} & $\begin{array}{l}\text { Interacción mediante las tecnologías digitales } \\
\text { Compartir información y contenidos digitales } \\
\text { Participación ciudadana en línea } \\
\text { Colaboración mediante canales digitales } \\
\text { Netiqueta }\end{array}$ \\
\hline & & Gestión de la identidad digital \\
\hline \multirow[t]{2}{*}{ Área 3} & \multirow[t]{2}{*}{$\begin{array}{l}\text { Creación de } \\
\text { contenidos } \\
\text { digitales }\end{array}$} & $\begin{array}{l}\text { Desarrollo de contenidos digitales } \\
\text { Integración y reelaboración de contenidos } \\
\text { digitales } \\
\text { Derechos de autor y licencias }\end{array}$ \\
\hline & & Programación \\
\hline \multirow[t]{2}{*}{ Área 4} & \multirow[t]{2}{*}{ Seguridad } & $\begin{array}{l}\text { Protección de los dispositivos } \\
\text { Protección de datos personales e identidad } \\
\text { digital } \\
\text { Protección de la salud }\end{array}$ \\
\hline & & Protección del entorno \\
\hline \multirow[t]{2}{*}{ Área 5} & \multirow[t]{2}{*}{$\begin{array}{l}\text { Resolución de } \\
\text { problemas }\end{array}$} & $\begin{array}{l}\text { Resolución de problemas técnicos } \\
\text { Identificación de necesidades y respuestas } \\
\text { tecnológicas } \\
\text { Innovación y uso de la tecnología digital de } \\
\text { forma creativa }\end{array}$ \\
\hline & & $\begin{array}{l}\text { Identificación de lagunas en la competencia } \\
\text { digital }\end{array}$ \\
\hline
\end{tabular}

Fuente: elaboración propia con base en lo propuesto por el Instituto Nacional de Tecnologías Educativas y de Formación del Profesorado (INTEF, 2017). 
Una opción a tener en cuenta para poder promover el fortalecimiento de la competencia digital docente es la proliferación de un perfil de docente que destaca por encima del resto a nivel tecnológico (Cuevas y Díaz, 2005; Leithwood y Seashore, 2011; Lorenzo, 2005; Ortega, 2012; Spillane, Camburn y Pareja, 2009) y que se erige como auténtico líder dinamizador de los procesos comunicativos y generador de un ambiente positivo entre el profesorado (Furguerle y Vitorá, 2016; Gayán, 2013; Gómez y Medina, 2014; Moral y Amores, 2014). Los líderes docentes serán los encargados de fomentar un cambio de actitud en aquellos docentes arraigados a la enseñanza tradicional y que muestran mayor desconfianza e inseguridad antes las nuevas corrientes pedagógicas centradas en la tecnología (Contreras, 2016).

En definitiva, los recursos digitales que se disponen en los centros educativos no son aprovechados de forma efectiva debido a la baja formación del profesorado (Santiago, Navaridas y Andía, 2016), e incluso en los casos en los que las TIC se encuentran incorporados en los centros, su inclusión en las metodologías didácticas no se produce de forma óptima y efectiva (Gisbert, González y Esteve, 2016). Por ello, en un panorama actual en el que la competencia digital docente ha cobrado una importancia capital al contribuir al hecho de que los docentes puedan aprovechar las potencialidades de la tecnología (Tejada y Pozos, 2018), resultará pertinente la existencia de un líder electrónico que se encargue de dinamizar e impulsar la utilización de las TIC en los centros educativos para que puedan ser incorporadas en el proceso de enseñanza y aprendizaje de forma eficaz.

Por otra parte, dentro del amplio abanico de tipología de centros educativos existentes en el panorama estatal, nos encontramos con un tipo de centro concreto: las cooperativas de enseñanza. Esta tipología de centro educativo se crea con base en los principios de la economía social para crear una oferta laboral para el profesorado (López, Moreno y Pozo, 2018), De esta forma, los integrantes de las cooperativas de enseñanza ejercen, por un lado, la labor docente, y por otro, una tarea de emprendimiento que se articula en un proyecto educativo innovador y eficiente (Fuentes, Lorenzo y Corchon, 2002; Fernández, 2003; Fuentes, 2004; Inglada, Sastre y Villarroya, 2015) con un importante componente de liderazgo entre los docentes que lo 
componen, destacando un perfil femenino en el desarrollo de este fenómeno (López, 2017; López y Fuentes, 2018).

Las cooperativas de enseñanza se erigen, por ende, como lugares propicios para la dinamización del proceso de enseñanza y aprendizaje con base en la figura de un líder electrónico que se encargue de fomentar el entusiasmo hacia los dispositivos tecnológicos y los espacios digitales de aprendizaje (Sosa y Valverde, 2015), consiguiendo que la competencia digital del profesorado que conforma la plantilla de esta tipología de centros se potencie y desemboque en una utilización cotidiana, efectiva y crítica de la tecnología (Rodríguez-García, Martínez y Raso, 2017), para responder eficazmente a las demandas del alumnado actual.

\section{Método}

\section{Justificación y delimitación contextual}

Las peculiaridades de la sociedad del presente hacen reflexionar al profesorado acerca de las prácticas efectuadas en el aula, con la intención de comprobar si realmente se adecuan a los nuevos entornos y contribuyen a satisfacer las necesidades de los alumnos actuales. Como se ha presentado anteriormente, la ciudadanía se encuentra inmersa en una era digital que ha condicionado a la educación (Cabero, 2017; González, 2017; Viñals y Cuenca, 2016). Es por ello que el proceso de aprendizaje debe adecuarse a los requerimientos e innovaciones que trae consigo la sociedad (Aróstegui y Guerrero, 2014; McCarthy, 2016; Vaillant, Rodríguez y Bernasconi, 2017).

Esta investigación tiene su razón de ser en la importancia de emplear nuevas praxis de carácter innovador en los procesos de enseñanza y aprendizaje dadas las singularidades de una época marcada por la incidencia tecnológica sobre las personas y la sociedad en general. Siendo fundamental para ello una formación continua en el tiempo por parte del docente — para adquirir las competencias necesarias que permitan desempeñar nuevas metodologías de trabajo en el entorno escolar - y la dinamización de todo el profesorado por parte de diversos e-líderes con la intención de fomentar y promover una transformación y evolución de los recursos didácticos utilizados hasta el momento. 
El estudio llevado a cabo se ha centrado en la región sur de España, singularmente en la Comunidad Autónoma de Andalucía, abarcando distintas provincias que la integran. En el sistema educativo español se conciben tres tipos de centros escolares. Ordenados según su proliferación se encuentran los centros públicos, seguidos en menor cifra por los privados y en una situación destacada por su escaso volumen se hallan las cooperativas de enseñanza, definidas como centros educativos caracterizados por el desarrollo de proyectos educativos innovadores con el propósito de ofertar una enseñanza sustentada en los principios de la calidad, innovación y excelencia, por medio del liderazgo y formación de índole multidisciplinar de su claustro docente (López, Fuentes y Moreno, 2018; López, Moreno y Pozo, 2018).

En concreto, el contexto de la investigación se ha focalizado en las cooperativas de enseñanza de distintas provincias que conforman la Comunidad Autónoma de Andalucía (España).

\section{Objetivo}

Una vez justificado el estudio y delimitado el contexto donde se desarrolla la investigación, se pretende como objetivo general conocer la incidencia del eliderazgo del docente transmitido por medio de grupos de trabajo para el desarrollo de nuevas prácticas innovadoras en los procesos de enseñanza y aprendizaje.

A raíz de este objetivo general se han desglosado las siguientes preguntas de investigación: ¿cuál es el perfil social del profesorado que integra las cooperativas de enseñanza?, ¿existen e-líderes en las cooperativas de enseñanza?, ¿cuál es el grado de formación continua de los docentes?, ¿qué tipo de metodologías docentes basadas en las TIC utiliza el profesorado?, ¿cuál es el nivel de competencia digital docente en las cinco áreas estipuladas en la legislación educativa vigente?, ¿influye el sexo de los docentes en el eliderazgo?, ¿influye el sexo de los docentes en la competencia digital? 
REMIE - Multidisciplinary Journal of Educational Research, 9(2) 203

\section{Diseño de investigación}

Se ha establecido un diseño de investigación de carácter cuantitativo, de tipo no experimental, siguiendo un tratamiento estadístico de naturaleza descriptiva y correlacional (McMillan y Schumacher, 2005), para dar respuesta al objetivo formulado.

\section{Variables definidas}

Las variables que se han utilizado en este estudio son el sexo del profesorado (SEX), su edad (AGE), sus años de experiencia docente (EXPE), su titulación académica (TITUL), la influencia que puede ejercer sobre los demás (INFLU), su capacidad de dinamización del colectivo (DINAM), su habilidad para captar seguidores (SEGUID), el número de cursos de formación continua (NCUR), la modalidad de los cursos realizados (MOD) y la temática formativa (TEMFOR), las metodologías utilizadas en los procesos de enseñanza-aprendizaje (METOD) y la competencia digital docente en cada una de las áreas (Ấrea 1 = CDD-1; Área 2 = CDD-2; Área 3 = CDD-3, Área 4 = CDD-4; Área 5 = CDD-5).

\section{Integrantes de la muestra}

Los sujetos que han participado en este estudio son docentes $(n=428)$ que desempeñan su cometido en diferentes etapas educativas en cooperativas de enseñanza de Andalucía (España). Los participantes han sido escogidos por medio de un muestreo intencional o de conveniencia, por causa de su eficaz acceso a la composición de una muestra representativa (Hernández, Fernández y Baptista, 2014). Toda la información necesaria para contactar con las distintas cooperativas de enseñanza fue extraída de la base de datos que contiene la página web (http://aces-andalucia.es/centros/) de la Asociación Andaluza de Centros de Enseñanza de la Economía Social (ACES). A continuación, en la tabla 2 se recogen los detalles acerca de las singularidades de la muestra de profesorado que han permitido desarrollar esta investigación. 
Tabla 2:

Muestra de participantes

\begin{tabular}{lll}
\hline Distribución por sexo & & \\
\hline & $\mathrm{N}$ & $\%$ \\
\hline Hombre & 197 & 46.03 \\
Mujer & 231 & 53.97 \\
\hline Distribución por edad & & \\
\hline $20-30$ años & 92 & 21.49 \\
$31-40$ años & 102 & 29.45 \\
$41-50$ años & 126 & 23.83 \\
Más de 50 años & 108 & 25.23 \\
\hline Experiencia laboral & & \\
\hline 1-10 años & 116 & 27.1 \\
$11-20$ años & 109 & 25.47 \\
$21-30$ años & 135 & 31.54 \\
Más de 30 años & 68 & 15.88 \\
\hline Titulación académica & & \\
\hline Diplomatura & 191 & 44.62 \\
\hline Grado & 53 & 12.38 \\
\hline Licenciatura & 157 & 36.68 \\
\hline Doctorado & 27 & 6.31 \\
\hline Total & $\mathbf{4 2 8}$ & $\mathbf{1 0 0}$ \\
\hline
\end{tabular}

\section{Instrumento}

La recogida de datos se ha efectuado a través de un cuestionario de naturaleza ad hoc, con la intención de configurar los diversos ítems para recoger la información necesaria que permita dar respuesta a las distintas variables presentadas (Alaminos y Castejón, 2006). Para medir el e-liderazgo del profesorado y la competencia digital de tales docentes se han tomado como referentes otros instrumentos previamente validados como el de García (2016) para valorar las prácticas de liderazgo y el de Tourón, Martín, 
Navarro, Pradas y Íñigo (2018) para medir la competencia digital del profesorado.

El instrumento utilizado se compone de 67 cuestiones que han sido clasificadas en diferentes dimensiones: a) Sociodemográfica (SD): 10 ítems; b) Liderazgo (LD): 18 ítems; c) Formación continua (FC): 8 ítems; d) Desempeño profesional (DP): 11 ítems; e) Competencia digital docente (CDD): 20 ítems.

Cada uno de los ítems formulados sigue - en su mayoría - un formato de respuesta en escala Likert de rango 1-4, donde uno es la valoración más negativa y cuatro la más positiva. Además de otras cuestiones con respuesta establecida, siendo su elección cerrada.

Diseñado el cuestionario se sometió a evaluación por medio de un juicio de expertos (Escobar y Cuervo, 2008) conformado por seis doctores especialistas en el área de conocimiento quienes procedieron a validar el contenido del instrumento con la intención de optimizar la herramienta a través del feedback otorgado por cada experto. Tras esta validación cualitativa, se inició el Análisis de Componentes Principales (ACP), una técnica de análisis multivariante que permite reducir los datos sin suprimir información. Ello se llevó a cabo con el test de Kaiser-Meyer-Olkin (KMO: $p=0.918)$, y el test de esfericidad de Bartlett $(p=0.0005)$, revelando una pertinencia en el ACP. Asimismo, se aplicó una rotación Promax de naturaleza oblicua con normalización Kaiser con el propósito de favorecer la interpretabilidad de los datos (Pérez, 2005).

Para conocer el grado de fiabilidad del cuestionario se calculó el Alfa $(\alpha)$ de Cronbach, revelando un $\alpha=0.855$ en la totalidad del instrumento. Arrojando los siguientes valores en cada una de las dimensiones establecidas: SD- $\alpha=0.816$; LD- $\alpha=0.847$; FC- $\alpha=0.835$; DP- $\alpha=0.881$; CDD- $\alpha=0.894$. Los valores hallados de $\alpha$ reflejan una alta consistencia interna de los ítems establecidos en el instrumento, al alcanzar valores comprendidos entre 0.8-1 (Bisquerra, 2004).

\section{Procedimiento}

A comienzos del curso académico 2017-2018 tuvo origen el estudio que se presenta en este trabajo de investigación. La primera acción efectuada fue 
establecer contacto con las cooperativas de enseñanza halladas en la Comunidad Autónoma de Andalucía, bajo la premisa de conocer su disponibilidad y aceptación en participar en un estudio de tal envergadura. Una vez aceptada la propuesta por distintos centros educativos, se enviaron por correo electrónico las instrucciones para la confección de grupos de trabajo que permitieran expandir todo el ideario que traen de manera implícita las TIC. En cada centro participante se formó un equipo docente liderado por un coordinador, con la finalidad de fomentar la formación docente del profesorado y renovar las metodologías didácticas.

Se estableció una temporalización de un curso académico para lograr alcanzar dicho objetivo planteado. Durante ese plazo, se efectuó un seguimiento mensual para conocer la evolución de los diversos grupos de trabajo, estableciendo un contacto directo con el coordinador de cada grupo. Transcurrido ese tiempo, todos los participantes de la muestra cumplimentaron el cuestionario aportado por los investigadores, cuyos datos han resultado de gran valía para continuar con la investigación.

\section{Análisis de datos}

Se ha utilizado como software de análisis el Statistical Package for the Social Sciences (SPSS) v.22, estableciendo un $p<0.05$ como diferencia estadísticamente significativa, con un elevado nivel de confianza $(Z=1.96)$ y un escaso margen de error $(e=0.05)$. En el análisis se han empleado estadísticos como la media $\left(\mathrm{M}_{\mathrm{e}}\right)$, la desviación típica (DT), el coeficiente de asimetría de Pearson $\left(\mathrm{CA}_{\mathrm{P}}\right)$ y el coeficiente de apuntamiento de Fisher $\left(\mathrm{CA}_{\mathrm{F}}\right)$. Y para la comparación de variables se han llevado a cabo las pruebas Chicuadrado $\left(\chi^{2}\right)$ y V de Cramer $\left(\mathrm{V}_{\text {Cramer }}\right)$, con base en las orientaciones de los expertos (Landero y González, 2006).

\section{Resultados}

En adelante, se muestran los resultados alcanzados en el estudio de las distintas variables que articulan la investigación. Comenzando por las variables que presentan una relación directa con el fenómeno del liderazgo, la tabla 3 determina que los docentes presentan valoraciones que se 
encuentran por encima de la media, puesto que el estadístico en las tres variables analizadas arroja un puntaje $>2$, siendo estos muy similares, destacando levemente la influencia que ejercen sobre los demás profesionales del centro educativo al que pertenecen los docentes.

Asimismo, para conocer el grado de asimetría de la distribución, se han efectuado los estadísticos $\mathrm{CA}_{\mathrm{P}}$ y $\mathrm{CA}_{\mathrm{F}}$, hallándose una asimetría positiva en los datos obtenidos con una tendencia de estos hacia la izquierda, produciéndose un grado de apuntamiento de tipo platicúrtico en la distribución presentada.

Tabla 3:

Resultados obtenidos en la dimensión liderazgo (LD)

\begin{tabular}{|c|c|c|c|c|c|c|c|c|}
\hline & \multicolumn{4}{|c|}{ Escala Likert $n(\%)$} & \multicolumn{4}{|c|}{ Parámetros } \\
\hline & Nada & Poco & Bastante & Totalmente & $\mathbf{M}_{\mathbf{e}}$ & DT & $\begin{array}{l}\mathbf{C A} \\
\mathbf{P}\end{array}$ & $\begin{array}{l}\mathbf{C A} \\
\mathrm{F}\end{array}$ \\
\hline INFLU & $\begin{array}{l}83 \\
(19.39)\end{array}$ & $\begin{array}{l}122 \\
(28.5)\end{array}$ & $134(31.3)$ & 89 (20.79) & 2.53 & $\begin{array}{l}1.02 \\
5\end{array}$ & $\begin{array}{l}1.49 \\
6\end{array}$ & $\begin{array}{l}- \\
1.12 \\
9\end{array}$ \\
\hline $\begin{array}{l}\text { DINA } \\
\text { M }\end{array}$ & $\begin{array}{l}102 \\
(23.83)\end{array}$ & $\begin{array}{l}147 \\
(34.34)\end{array}$ & $116(27.1)$ & 63 (14.72) & 2.32 & $\begin{array}{l}0.99 \\
5\end{array}$ & $\begin{array}{l}1.33 \\
3\end{array}$ & $\begin{array}{l}- \\
1.01 \\
3\end{array}$ \\
\hline $\begin{array}{l}\text { SEGUI } \\
\text { D }\end{array}$ & $\begin{array}{l}91 \\
(21.26)\end{array}$ & $\begin{array}{l}142 \\
(33.17)\end{array}$ & $\begin{array}{l}138 \\
(32.24)\end{array}$ & 57 (13.32) & 2.37 & $\begin{array}{l}0.96 \\
2\end{array}$ & $\begin{array}{l}1.42 \\
9\end{array}$ & $\begin{array}{l}- \\
0.96 \\
7\end{array}$ \\
\hline
\end{tabular}

Nota: INFLU = influencia que puede ejercer sobre los demás; DINAM = capacidad de dinamización del colectivo; SEGUID = habilidad para captar seguidores.

La figura 1 contiene distintas variables analizadas en la dimensión formación continua (FC). Con respecto a los cursos TIC por año, se obtiene que aproximadamente — casi la mitad de los participantes — no realizan más de un curso de formación continua al año. En cuanto a la temática de estos, se arrojan valoraciones similares entre aquellos docentes que llevan a cabo cursos genéricos sobre tecnología educativa que no siguen una temática en concreto y otros profesionales que no efectúan cursos relacionados con las TIC. Sobre la modalidad formativa, se ha obtenido que exactamente la mitad de los docentes encuestados optan por seguir una modalidad formativa 
presencial, seguida — en menor grado - por una a distancia mediada por la tecnología.

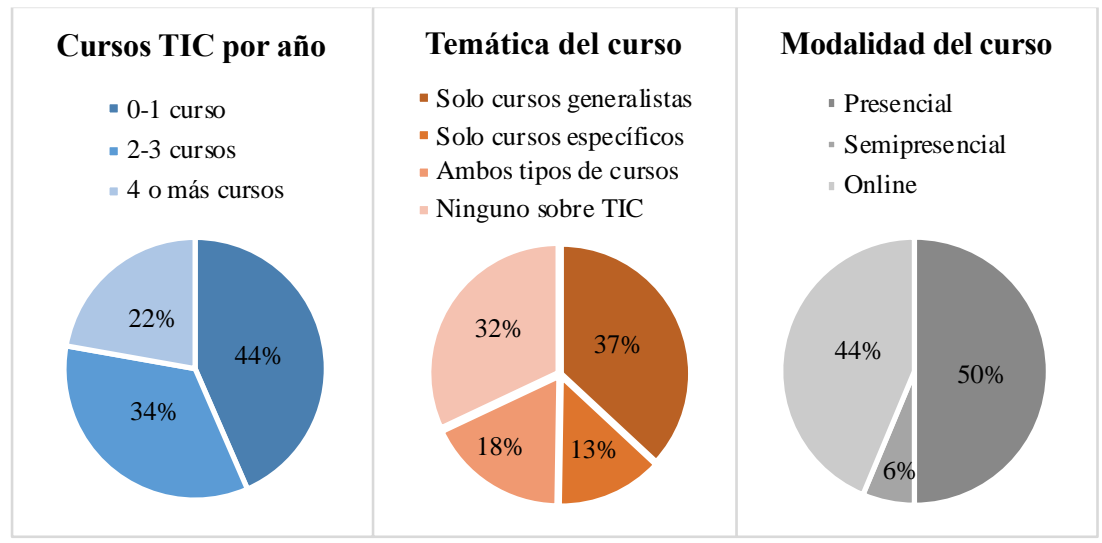

Figura 1. Resultados alcanzados en la formación del profesorado

Tras la puesta en marcha del grupo de trabajo, la figura 2 revela que los docentes han desplegado, por un lado, metodologías combinadas en las que el docente ha transmitido los contenidos de manera expositiva, pero se ha apoyado en el uso de las TIC y, por otro, metodologías tradicionales fundamentadas en la mera reproducción de contenidos por parte del profesorado y actitud pasiva y receptora del discente. Aunque - muy de cerca- le sigue un grupo de profesionales que han llevado a cabo metodologías focalizadas principalmente en las TIC. También se visualiza, en menor cifra, un colectivo docente que aboga por la escasez de uso de la tecnología educativa.

Sobre la competencia digital docente revelada por los participantes, la tabla 4 muestra las puntuaciones obtenidas en cada una de las áreas que conforman la competencia digital de los participantes analizados. Aunque se han alcanzado valores por encima de la media, excepto en el área concerniente a la creación de contenidos digitales (CDD-3), se aprecia un ligero despunte en las áreas una, dos y cinco, correspondientes a las destrezas en información y alfabetización informacional, comunicación-colaboración y resolución de problemas, respectivamente. 
$\mathrm{Al}$ igual que ha sucedido en la dimensión de liderazgo, las pruebas CAP y $\mathrm{CA}_{\mathrm{F}}$ han determinado que los datos contenidos en la dimensión competencia digital docente (CDD) producen una positividad asimétrica de la distribución con inclinación hacia la izquierda, dando lugar a un fenómeno platicúrtico en el apuntamiento.

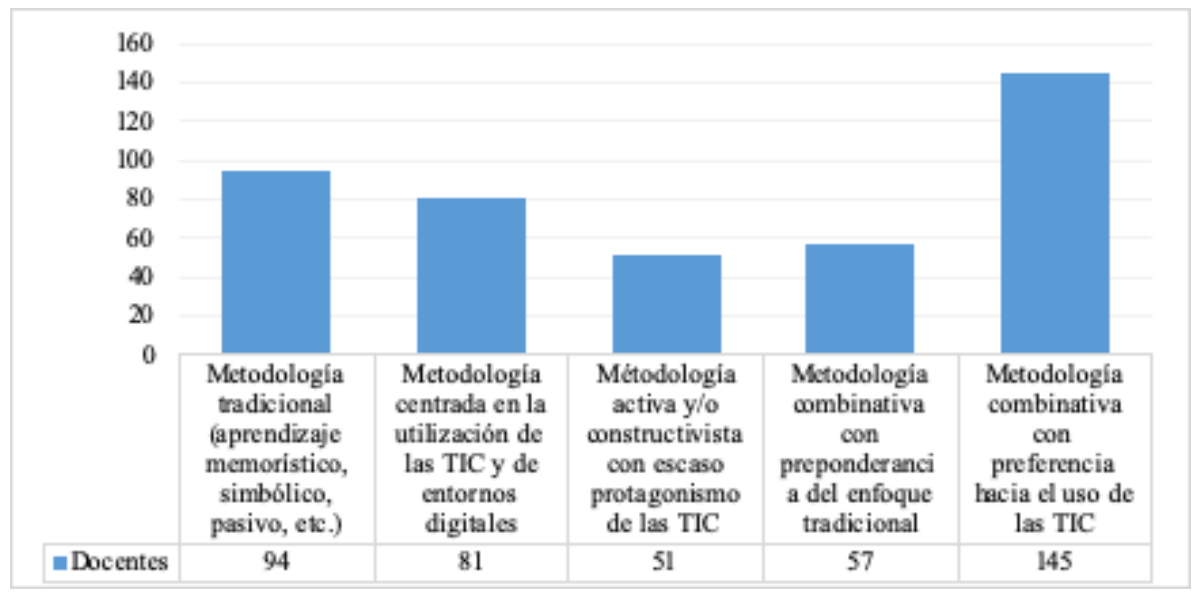

Figura 2. Resultados alcanzados en la metodología utilizada

Tabla 4:

Resultados arrojados en la dimensión competencia digital docente (CDD)

\begin{tabular}{lllllllll}
\hline & \multicolumn{1}{l}{ Escala Likert $n(\%)$} & & \multicolumn{5}{c}{ Parámetros } \\
\cline { 2 - 9 } & Nada & Poco & Bastante & Totalmente & Me & DT & CAP & CAF \\
CDD-1 & $43(10.04)$ & $81(18.9)$ & $123(28.73)$ & $181(42.28)$ & 3.032 & 1.006 & 2.019 & -0.757 \\
CDD-2 & $45(10.51)$ & $62(14.48)$ & $191(44.62)$ & $130(30.37)$ & 2.948 & .931 & 2.093 & -.331 \\
CDD-3 & $204(47.66)$ & $130(30.37)$ & $63(14.72)$ & $31(7.24)$ & 1.815 & .937 & .869 & -.219 \\
CDD-4 & $91(21.26)$ & $102(23.83)$ & $123(28.73)$ & $112(26.16)$ & 2.598 & 1.09 & 1.465 & -1.277 \\
CDD-5 & $58(13.55)$ & $75(17.52)$ & $151(35.28)$ & $144(33.64)$ & 2.890 & 1.021 & 1.852 & -.833 \\
\hline
\end{tabular}

Nota: Área 1 = CDD-1; Área 2 = CDD-2; Área 3 = CDD-3, Área 4 = CDD-4; Área 5 = CDD-5. 
Para concretar la influencia de la variable sexo sobre el liderazgo manifestado por los sujetos en el ámbito tecnológico, se ha recurrido a la aplicación del estadístico Chi-cuadrado de Pearson. Como se aprecia en la siguiente tabla 5, la prueba ha reportado diferencias estadísticamente significativas en la dinamización del colectivo $\left[\chi^{2}(3)=11.79, p<0.05\right]$ y en la obtención de seguidores $\left[\chi^{2}(3)=10.47, p<0.05\right]$ dentro de la entidad escolar, con predominancia de las mujeres en el fenómeno del liderazgo. Asimismo, para conocer el tamaño del efecto producido en tales variables, se ha realizado el test $\mathrm{V}_{\text {Cramer }}$, revelando debilidad en su asociación al obtener valores $<0.2$.

Tabla 5:

Asociación entre el sexo docente y el e-liderazgo

\begin{tabular}{llllll}
\hline & \multicolumn{3}{l}{ Sexo $n(\%)$} & \multicolumn{3}{c}{ Parámetros } \\
\cline { 2 - 6 } Likert & Hombre & Mujer & $\chi^{2}(\boldsymbol{g l})$ & $\begin{array}{l}\boldsymbol{p} \text { - } \\
\text { valor }\end{array}$ & $\mathbf{V}_{\text {Cramer }}$ \\
INFLU & & & $0.97(3)$ & .808 & .048 \\
Nada & $36(8.41)$ & $47(10.98)$ & & & \\
Poco & $56(13.08)$ & $66(15.42)$ & & & \\
Bastante & $66(15.42)$ & $68(15.88)$ & & & \\
Totalmente & $39(9.11)$ & $50(11.68)$ & & & \\
DINAM & & & & & \\
Nada & $55(12.85)$ & $47(10.98)$ & & $\mathbf{0 . 0 0 8}$ & $\mathbf{0 . 1 6 6}$ \\
Poco & $74(17.29)$ & $63(14.72)$ & & & \\
Bastante & $50(11.68)$ & $66(15.42)$ & & & \\
Totalmente & $18(4.2)$ & $45(10.51)$ & & & \\
SEGUID & & & & & \\
Nada & $32(7.47)$ & $58(13.55)$ & & & \\
Poco & $60(14.02)$ & $82(19.16)$ & & & \\
Bastante & $72(16.82)$ & $66(15.42)$ & & & \\
Totalmente & $33(7.71)$ & $24(5.61)$ & & & \\
\hline
\end{tabular}

Nota: INFLU = influencia que puede ejercer sobre los demás; DINAM = capacidad de dinamización del colectivo; SEGUID = habilidad para captar seguidores.

Para determinar la influencia entre el sexo de los docentes y la competencia digital, se ha desglosado esta última en las distintas áreas de que la componen y se ha efectuado la prueba Chi-cuadrado de Pearson para concretar si se hallan 
diferencias a nivel estadístico entre las variables presentadas en la tabla 6. La prueba ha arrojado altos niveles de significancia estadística en las variables relacionadas con la tenencia de destrezas en comunicación-colaboración, creación de contenidos y seguridad, dado los valores de $p<0.05$, con una fuerza de asociación moderada en el tamaño del efecto en CDD-2 y CDD-4 con base en los valores de $0.2<\mathrm{V}_{\text {Cramer }}<0.6$, mientras que en CDD-3 la fuerza obtenida es débil $\left(\mathrm{V}_{\text {Cramer }}<0.2\right)$.

Tabla 6:

Asociación entre el sexo docente y la competencia digital

\begin{tabular}{|c|c|c|c|c|c|}
\hline & $\operatorname{Sexo} n(\%)$ & & Parámetro & & \\
\hline Likert & Hombre & Mujer & $\chi^{2}(g l)$ & $p$-valor & $V_{\text {Crame }}$ \\
\hline CDD-1 & & & $0.76(3)$ & 0.859 & 0.042 \\
\hline Nada & $21(4.9)$ & $22(5.14)$ & & & \\
\hline Poco & $39(9.11)$ & $42(9.81)$ & & & \\
\hline Bastante & $58(13.55)$ & 65 (15.18) & & & \\
\hline Totalmente & 79 (18.46) & $102(23.83)$ & & & \\
\hline CDD-2 & & & $23.08(3)$ & $<0.001$ & 0.226 \\
\hline Nada & $35(8.17)$ & $10(2.33)$ & & & \\
\hline Poco & $32(7.47)$ & $30(7.01)$ & & & \\
\hline Bastante & $76(17.75)$ & $115(26.87)$ & & & \\
\hline Totalmente & $54(12.61)$ & $76(17.75)$ & & & \\
\hline CDD-3 & & & $12.04(3)$ & 0.007 & 0.165 \\
\hline Nada & 80 (18.69) & $124(28.97)$ & & & \\
\hline Poco & $61(14.25)$ & $69(16.12)$ & & & \\
\hline Bastante & 35 (8.17) & $28(6.54)$ & & & \\
\hline Totalmente & $21(4.9)$ & $10(2.33)$ & & & \\
\hline CDD-4 & & & $25.71(3)$ & $<0.001$ & 0.238 \\
\hline Nada & $35(8.17)$ & $56(13.08)$ & & & \\
\hline Poco & $30(7.01)$ & $72(16.82)$ & & & \\
\hline Bastante & 63 (14.72) & $60(14.02)$ & & & \\
\hline Totalmente & $69(16.12)$ & $43(10.04)$ & & & \\
\hline CDD-5 & & & $1.94(3)$ & 0.585 & 0.067 \\
\hline Nada & $23(5.37)$ & $35(8.17)$ & & & \\
\hline Poco & 34 (7.94) & $41(9.58)$ & & & \\
\hline Bastante & $68(15.88)$ & 83 (19.39) & & & \\
\hline Totalmente & $72(16.82)$ & $72(16.82)$ & & & \\
\hline
\end{tabular}

Nota: Área 1 = CDD-1; Área 2 = CDD-2; Área 3 = CDD-3, Área 4 = CDD-4; Área 5 = CDD-5. 


\section{López, Pozo, Fuentes, \& Romero-Liderazgo Digital}

\section{Discusión y Conclusiones}

Aunque los datos muestran un panorama optimista en cuanto a la adquisición y dominio de la competencia digital, se encuentran algunas deficiencias en torno a la formación recibida y las cualidades para ejercer realmente un liderazgo electrónico. Así pues, en relación a las variables que componen la dimensión de liderazgo, la mejor valorada por más de la mitad del profesorado es la influencia que ejercen sobre los demás (INFLU). Por ende, la mayor parte de los docentes creen que poseen un cierto grado de influencia sobre el resto, pudiendo ser potenciales e-líderes (Pozuelo, 2014). Sin embargo, se invierte el porcentaje de acuerdo a la habilidad para dinamizar (DINAM) y fomentar una actitud positiva en los compañeros o captar seguidores (SEGUID), lo cual es necesario para ejercer el liderazgo (Macías et al., 2018). Estas habilidades son clave para ser un auténtico líder dinamizador que genere un ambiente positivo (Furguerle y Vitorá, 2016; Gayán, 2013; Gómez y Medina, 2014; Moral y Amores, 2014).

No obstante, en las variables DINAM y SEGUID se obtienen diferencias estadísticamente significativas en función del sexo, siendo la media superior en las mujeres en cuanto a la dinamización y superior en los hombres en relación a la captación de seguidores.

Por otro lado, el esfuerzo por incluir las TIC en los centros educativos no se ve totalmente reflejado, puesto que el profesorado presenta una formación escasa en TIC (Area, Hernández y Alonso, 2016; Fernández, Fernández y Rodríguez, 2018; Santiago, Navaridas y Andía, 2016). Sin embargo, hay cierta motivación por formarse en materia tecnológica, reflejada en los docentes que acuden a este tipo de formación específica, los cuales valoran esta formación como crucial para su desempeño profesional (Kumar y Kumar, 2018; McGill, Koppi y Armarego, 2014; Murillo, Garrido y Hernández, 2016; Vacek y Rybenska, 2015).

Por su parte, predomina la combinación metodológica, es decir, presencialidad y TIC, lo cual concuerda con la dinamización del alumnado y el cambio del rol pasivo a activo (Cerda y Saiz, 2018; Jovanović et al., 2017; Sola et al., 2019). En menor medida, todavía hay algunos docentes arraigados únicamente en una metodología tradicional (Contreras, 2016).

En general, el profesorado presenta un grado no uniforme en su competencia digital docente (Blanco, 2018; INTEF, 2017). Asimismo, en las áreas 1 (Información y alfabetización digital), 2 (Comunicación y colaboración) y 5 (Resolución de problemas) podrían situarse en torno al nivel C (superior), 
mientras que el área 4 (Seguridad) podría situarse en el nivel B (medio) y el área 3 (Creación de contenidos digitales) en el nivel A (inicial) (Durán, Gutiérrez y Prendes, 2016; Esteve, 2016).

Esto es un posible indicador de la falta de formación docente en TIC, puesto que aquellas áreas que requieren de un nivel superior de competencia digital obtienen la puntuación más baja (Martínez, 2018; Moreno, Gabarda y Rodríguez, 2018; Peirats et al., 2018).

A diferencia del estudio realizado por Fernández y Fernández (2016), si atendemos a las diferencias de sexo, se obtienen puntuaciones estadísticamente significativas las áreas 2,3 y 4, a favor de las mujeres en el área 2 y con medias ligeramente superiores por parte de los hombres en las áreas 3 y 4 . Estas diferencias pueden arrojar posibles preferencias a la hora del uso de la tecnología, puesto que el área 2 se corresponde con su uso para la comunicación y colaboración y la 3 y 4 con la creación de contenidos y el uso seguro de la red. En suma, el estudio sigue la línea de trabajos anteriores, centrados en el análisis de la competencia digital, en el nivel que presenta el profesorado en tal competencia profesional y en la formación en TIC (Falcó, 2017; Fernández y Fernández, 2016; González y Gutiérrez, 2017; Pérez y Rodríguez, 2016; Rodríguez-García, Cáceres y Alonso, 2018).

Los datos obtenidos contrastan con el hecho de que las cooperativas de enseñanza se basen en proyectos educativos innovadores (Fuentes, Lorenzo y Corchón, 2002; Fernández, 2003; Fuentes, 2004; Inglada, Sastre y Villarroya, 2015). Esto se debe — en primera instancia - a la falta de competencia digital en niveles superiores y en el hecho de no poseer las cualidades necesarias para ejercer un auténtico e-liderazgo.

Teniendo en cuenta estas consideraciones, se ha dado respuesta tanto al objetivo planteado acerca de conocer la incidencia del liderazgo electrónico de los docentes de las cooperativas de enseñanza de Andalucía, como a las preguntas de investigación planteadas. De modo que se ha establecido el perfil social del profesorado que integra las cooperativas de enseñanza, en su mayoría mujeres con una edad superior a los 41 años. Se ha determinado la escasez de elíderes en las cooperativas de enseñanza, puesto que mayormente no cumplen con todas las cualidades requeridas (INFLU, DINAM y SEGUID). Se ha definido la formación continua de los docentes en materia tecnológica, la cual es baja, así como el tipo de metodología aplicada, que en su mayoría combina la presencialidad con las TIC. Se ha fijado el nivel de competencia digital de los 


\section{López, Pozo, Fuentes, \& Romero-Liderazgo Digital}

docentes en torno a las cinco áreas, destacando el alto nivel en las áreas 1, 2 y 5, medio en el área 4 y bajo en el área 3. Se han detectado diferencias estadísticamente significantes en función del sexo en las cualidades del e-líder y en ciertas áreas de la competencia digital docente.

Como prospectiva de futuro, hay que destacar la continuidad de trabajos que sigan la línea de estudio sobre la implementación de las TIC en las aulas, puesto que la tendencia en la aplicación de la tecnología va en aumento y debe realizarse un seguimiento del grado de preparación del profesorado para su correcto desempeño. Además, futuros estudios podrían abordar las necesidades formativas concretas que demanda el profesorado, las cuales a raíz de este trabajo podrían establecerse en torno al área 3 de la competencia digital: la creación de contenidos digitales. Asimismo, otra posible línea de estudio sería comprobar si el número de cursos de formación en TIC es proporcional al nivel de competencia digital del profesorado.

Finalmente, es necesario plantear estudios que manifiesten el interés por las TIC, la formación docente y las ventajas e inconvenientes de su implementación en el aula. Por tanto, se augura un panorama totalmente digitalizado, donde el rol docente va a jugar un papel fundamental para discernir y educar en un buen uso de la tecnología.

\section{Referencias}

Alaminos, A. y Castejón, J.L. (2006). Elaboración, análisis e interpretación de encuestas, cuestionarios y escalas de opinión. Alicante: Marfil.

Area, M. y Guarro, A. (2012). La alfabetización informacional y digital: fundamentos pedagógicos para la enseñanza y el aprendizaje competente. Revista española de documentación científica, 35, 46-74. doi: 10.3989/redc.2012.mono.977

Area, M., Hernández, V. y Alonso, J.J. (2016). Modelos de integración didáctica de las TIC en el aula. Comunicar: Revista científica iberoamericana de comunicación y educación, 24(47), 79-87. doi: 10.3916/C47-2016-08

Aróstegui, J.L. y Guerrero, J.L. (2014). El Papel de las TIC en la Mejora de la Calidad Docente en Secundaria: Un Estudio Multicasos. Multidisciplinary Journal of Educational Research, 4(1), 101-124. doi: 10.4471/remie.2014.04 
Aznar, I., Cáceres, M.P. y Romero, J.M. (2018). Indicadores de calidad para evaluar buenas prácticas docentes de «mobile learning» en Educación Superior. Education in the Knowledge Society (EKS), 19(3), 53-68. doi: 10.14201/eks20181935368

Bisquerra, R. (2004). Metodología de la investigación educativa. Madrid: La Muralla.

Blanco, M. (2018). Marco Común de Competencia Digital Docente. Revista Iberoamericana de Educación a Distancia, 21(1), 369-370.

Cabero, J. (2017). La formación en la era digital: ambientes enriquecidos por la tecnología. Revista Gestión de la Innovación en Educación Superior, 2(2), 41-64. Recuperado de: http://hdl.handle.net/11441/67192

Cerda, C. y Saiz, J.L. (2018). Aprendizaje autodirigido del saber pedagógico con tecnologías digitales. Generación de un modelo teórico en estudiantes de pedagogía chilenos. Perfiles educativos, 11(162), 138157. Recuperado de:

http://www.iisue.unam.mx/perfiles/descargas/pdf/2018-162-138-157

Comisión Europea (2009). Marco estratégico Educación y Formación 2020 (ET2020). España: Eurydice. Retrieved from: http://www.mecd.gob.es/educacion-mecd/mc/redieeurydice/prioridades-europeas/et2020.html

Comisión Europea (2016). Education and training Monitor 2016, Portugal. Retrieved from:

https://ec.europa.eu/education/sites/education/files/monitor2016pt_en.pdf

Contreras, T.S. (2016). Liderazgo pedagógico, liderazgo docente y su papel en la mejora de la escuela: una aproximación teórica. Propósitos y representaciones, 4(2), 231-284. doi: 10.20511/pyr2016.v4n2.123

Cuevas, M. y Díaz, F. (2005). El liderazgo educativo en centros de secundaria. Un estudio en el contexto multicultural de Ceuta. Revista Iberoamericana de Educación, 38(2), 1-17. doi: 10.35362/rie3722722

Díaz, D. (2013). TIC en Educación Superior: Ventajas y desventajas. Revista educación y tecnología, (4), 44-50. Recuperado de: https://dialnet.unirioja.es/servlet/articulo?codigo=5072156

Durán, M., Gutiérrez, I. y Prendes, M.P. (2016). Análisis conceptual de modelos de competencia digital del profesorado universitario. 
RELATEC: Revista Latinoamericana de Tecnología Educativa, 15(1), 97-114. doi: 10.17398/1695-288X.15.1.97

Escobar, J. y Cuervo, A. (2008). Validez de contenido y juicio de expertos: una aproximación a su utilización. Avances en medición, 6(1), 27-36.

Recuperado de:

http://www.humanas.unal.edu.co/psicometria/files/7113/8574/5708/Arti culo3_Juicio_de_expertos_27-36.pdf

Esteve, F. (2016). Bolonia y las TIC: de la docencia 1.0 al aprendizaje 2.0. La cuestión universitaria, 5, 58-67. Recuperado de:

http://polired.upm.es/index.php/lacuestionuniversitaria/article/view/3337

Falcó, J.M. (2017). Evaluación de la competencia digital docente en la Comunidad Autónoma de Aragón. Revista electrónica de investigación educativa, 19(4), 73-83. doi: 10.24320/redie.2017.19.4.1359

Fernández, F.J. y Fernández, M.J. (2016). Generation Z's Teachers and their Digital Skills. Comunicar, 24(46), 97-105. doi: 10.3916/C46-2016-10

Fernández, F.J., Fernández, M.J. y Rodríguez, J.M. (2018). El proceso de integración y uso pedagógico de las TIC en los centros educativos madrileños. Educación XX1, 21(2), 395-416. doi:

10.5944/educxx1.17907

Fernández, J. (2003). La participación, factor determinante de la calidad educativa de los centros docentes cooperativos. REVESCO: revista de estudios cooperativos, 79, 35-58.

Fernández, J. M. y Rodríguez, A. (2017). TIC y diversidad funcional: conocimiento del profesorado. EJIHPE. European Journal of Investigation in Health, Psychology and Education, 7(3), 157-175.

Recuperado de: https://hdl.handle.net/11441/69546

Fuentes, A. (2004). Las cooperativas de enseñanza: (un estudio de las cooperativas de trabajo asociado). Ceuta, España: Universidad Nacional de Educación a Distancia, Centro Asociado de Ceuta.

Fuentes, A., López, J. y Pozo, S. (2019). Análisis de la competencia digital docente: Factor clave en el desempeño de pedagogías activas con Realidad Aumentada. REICE. Revista Iberoamericana sobre Calidad, Eficacia y Cambio en Educación, 17(2), 27-42. doi: 10.15366/reice2019.17.2.002

Fuentes, A., Lorenzo, M. y Corchón, E. (2002). Las cooperativas de enseñanza como tercera vía dentro de nuestro sistema educativo: las cooperativas 
de trabajo asociado. Percepciones de sus directivos. Enseñanza, 20, 5184. Recuperado de: http://e-

spacio.uned.es/fez/eserv/bibliuned:20368/cooperativas_ensenanza.pdf

Furguerle, J. y Vitorá, M. (2016). Liderazgo en los directivos de educación primaria. Telos: Revista de Estudios Interdisciplinarios en Ciencias Sociales, 18(2), 208-227. Recuperado de:

http://www.redalyc.org/pdf/993/99345727004.pdf

Gallego, M.J., Gámiz, V. y Gutiérrez, E. (2010). El futuro docente ante las competencias en el uso de las tecnologías de la información y comunicación para enseñar, EDUTEC, Revista de Tecnología Educativa, 34, 1-18. doi: 10.21556/edutec.2010.34.418

García, M. (2016). Elaboración y validación de un cuestionario para medir prácticas eficaces de liderazgo pedagógico de la dirección. Profesorado. Revista de Currículum y Formación de Profesorado, 20(3), 493-526. Recuperado de: http://www.redalyc.org/pdf/567/56749100022.pdf Gayán, M. (2013). El liderazgo escolar según la LOMCE. Forum Aragón: revista digital de FEAE-Aragón sobre organización y gestión educativa, 7, 20-21. Recuperado de: https://dialnet.unirioja.es/descarga/articulo/4218610.pdf

Gisbert, M., González, J. y Esteve, F.M. (2016). Competencia digital y competencia digital docente: una panorámica sobre el estado de la cuestión. Revista Interuniversitaria de Investigación en Tecnología Educativa, 0, 74-83. doi: 10.6018/riite2016/257631

Gómez, R.M. y Medina, A. (2014). El liderazgo pedagógico: competencias necesarias para desarrollar un programa de mejora en un centro de educación secundaria. Perspectiva Educacional, 53(1), 91-113. doi: 10.4151/07189729

González, A. (2017). Dinamización tecnológica de la escuela a través del liderazgo del coordinador TIC. Estudios pedagógicos, 43(2), 115-125. doi: 10.4067/S0718-07052017000200006.

González, R. y Gutiérrez, A. (2017). Competencias mediática y digital del profesorado e integración curricular de las tecnologías digitales. Revista Fuentes, 19(2), 57-67. doi: 10.12795/revistafuentes.2016.19.2.04 Hernández, R., Fernández, C. y Baptista, P. (2014). Metodología de la investigación. Madrid: McGraw Hill. 
Inglada, M.E., Sastre, J.M. y Villarroya, M.B. (2015). El cooperativismo en la educación. REVESCO: Revista de estudios cooperativos, 1(118), 122147. Recuperado de: http://www.redalyc.org/pdf/367/36739634005.pdf

Instituto Nacional de Tecnologías Educativas y de Formación del Profesorado (INTEF) (2017). Marco de Competencia Digital. Madrid, España:

Ministerio de Educación, Ciencia y Deportes.

Jovanović, J., Gašević, D., Dawson, S., Pardo, A. y Mirriahi, N. (2017).

Learning analytics to unveil learning strategies in a flipped classroom. The Internet and Higher Education, 33(4), 74-85.

https://psycnet.apa.org/doi/10.1016/j.iheduc.2017.02.001

Kumar, A. y Kumar, G. (2018). The Role of ICT in Higher Education for the 21st Century: ICT as A Change Agent for Education. Multidisciplinary Higher Education, Research, Dynamics \& Concepts: Opportunities \& Challenges For Sustainable Development, 1(1), 76-83. Recuperado de: https://pdfs.semanticscholar.org/477b/db4337b5c997b54a66529861633c 03c737ad.pdf

Landero, R. y González, M. (2006), Estadística con SPSS y metodología de la investigación. México: Trillas.

Laskaris, D., Kalogiannakis, M. y Heretakis, E. (2017). Interactive evaluation of an e-learning course within the context of blended education.

International Journal of Technology Enhanced Learning, 9(4), 339-353.

Leithwood, K. y Seashore, K. (2011). Linking leadership to student learning. San Francisco: Jossey-Bass.

López, J. (2017). El fenómeno del liderazgo en los centros de enseñanza de naturaleza cooperativa. En J.M. Alcántara, M. Bermúdez, F.J. Blanco y J.M. Heredia (eds.), Investigación e innovación en el ámbito universitario. Tendencias ante los retos actuales de la sociedad (pp. 2746). Madrid, España: EOS.

López, J. y Fuentes, A. (2018). El liderazgo aplicado a los modelos diferenciados en educación: El caso de las cooperativas de enseñanza. En J. Gairín y C. Mercader (eds.), Liderazgo y gestión del talento en las organizaciones (pp. 169-175), Madrid, España: Wolters Kluwer.

López, J., Fuentes, A. y Moreno, A.J. (2018). El liderazgo efectivo en los centros concertados de naturaleza cooperativa: Percepciones de sus docentes. Revista actualidades investigativas en educación, 18(3), 1-21. 
López, J., Moreno, A.J. y Pozo, S. (2018). Influencia del género y la edad en la formación continua multidisciplinar de los docentes de cooperativas de enseñanza. Revista Innova, 3(8), 42-59. Recuperado de:

10.33890/innova.v3.n8.1.2018.756

López, M. y Bernal, C. (2019). El perfil del profesorado en la Sociedad Red: reflexiones sobre la competencia digital de los y las estudiantes en Educación de la Universidad de Cádiz. IJERI: International Journal of Educational Research and Innovation, 11, 83-100.

Lorenzo, M. (2005). El liderazgo en las organizaciones educativas: revisión y perspectivas actuales. Revista española de pedagogía. 63(232), 367-388. Recuperado de: https://dialnet.unirioja.es/descarga/articulo/1373221.pdf

Macías, E.J., Chum, S.R., Aray, C.A. y Rodríguez, C.J. (2018). Liderazgo academico: Estilos y perfiles de gestión en las instituciones de educación superior. ReHuSo: Revista de Ciencias Humanísticas y Sociales, 3(1), 82-105. doi: 10.18800/contabilidad.201701.009

Maquilón, J. J., Mirete, A. B. y Avilés, M. (2017). La Realidad Aumentada (RA). Recursos y propuestas para la innovación educativa. Revista Electrónica Interuniversitaria de Formación del Profesorado, 20(2), 183-204. doi: 10.6018/reifop/20.2.290971

Martín, A.V., García, A. y Muñoz, J.M. (2014). Factores determinantes de adopción de blended learning en educación superior. Adaptación del modelo UTAUT. Educación XX1, 17(2), 217-240. doi:

10.5944/educxx1.17.2.11489

Martínez, A. (2018). Percepciones sobre las competencias de formación básica en Educación Infantil, Aula de Encuentro, 20(1), 54-73. Recuperao de: https://revistaselectronicas.ujaen.es/index.php/ADE/article/download/36 $53 / \mathrm{pdf}$

Mccarthy, J. (2016). Reflections on a flipped classroom in first year higher education. Issues in Educational Research, 26(2), 332. Recuperado de: http://www.iier.org.au/iier26/mccarthy-j.pdf

McGill, T., Koppi, T. y Armarego, J. (2014). ICT industry involvement with ICT education and research in universities: Industry perceptions.

Innovation in Teaching and Learning in Information and Computer Sciences, O(0), 1-18. doi: 10.11120/ital.2014.00010

McMillan, J.H. y Schumacher, S. (2005). Investigación educativa. Madrid: Pearson. 
Méndez, J.M. y Delgado, M. (2016). Las TIC en centros de Educación Primaria y Secundaria de Andalucía. Un estudio de casos a partir de buenas prácticas. Digital Education Review, 29, 134-165. doi:

10.1344/der.2016.29.134-165

Mingorance, A. C., Trujillo, J. M., Cáceres, M. P. y Torres, C. (2017). Mejora del rendimiento académico a través de la metodología de aula invertida centrada en el aprendizaje activo del estudiante universitario deficiencias de la educación. Journal of Sport and Health Research, 9, 129-136. Recuperado:

http://www.journalshr.com/papers/Vol\%209_suplemento/JSHR\%20V09 supl_05.pdf

Moral, C. y Amores, F.J. (2014). Arquitectura resistente determinante de liderazgo pedagógico en los centros de Educación Secundaria. Bordón. Revista de pedagogía, 66(2), 121-138. Recuperado de: https://dialnet.unirioja.es/servlet/articulo?codigo $=4673894$

Moreno, M.D., Gabarda, V. y Rodríguez, A.M. (2018). Alfabetización informacional y competencia digital en estudiantes de magisterio. Profesorado, Revista de currículum y formación del profesorado, 22(3), 253-270. Recuperado de: https://dialnet.unirioja.es/servlet/articulo?codigo $=6026403$

Murillo, F.J., Garrido, C. y Hernández, R. (2016). Decálogo para una enseñanza eficaz. REICE. Revista Iberoamericana sobre Calidad, Eficacia y Cambio en Educación, 9(1), 7-27. Recuperado de: http://www.redalyc.org/pdf/551/55118790002.pdf

Ortega, E.R. (2012). Liderazgo y administración en la educación. Visión Educativa IUNAES, 6(13), 25-34. Recuperado de: https://dialnet.unirioja.es/descarga/articulo/3995940.pdf

Peirats, J., Marín, D., Granados, J. y Morote, D. (2018). Competencia digital en los planes de estudios de universidades públicas españolas. REDU: Revista de Docencia Universitaria, 16(1), 175-191. Recuperado de: https://polipapers.upv.es/index.php/REDU/article/download/8935/10306

Pérez, A. y Rodríguez, M.J. (2016). Evaluación de las competencias digitales autopercibidas del profesorado de Educación Primaria en Castilla y León (España). Revista de Investigación Educativa, 34(2), 399-415. doi: 10.6018/rie.34.2.215121 
REMIE - Multidisciplinary Journal of Educational Research, 9(2) 221

Pérez, C. (2005). Métodos estadísticos avanzados con SPSS. Madrid: Thomson.

Pozos, K. y Tejada, J. (2018), Competencias digitales docentes en educación superior: niveles de dominio y necesidades formativas. Revista Digital de Investigación en Docencia Universitaria (RIDU), 13(2), 59-87. doi: 10.19083/ridu.2018.71

Pozuelo, J. (2014). ¿Y si enseñamos de otra manera? Competencias digitales para el cambio metodológico. Caracciolos. Revista digital de investigación en docencia, 2(1), 1-21. Recuperado de:

https://ebuah.uah.es/dspace/bitstream/handle/10017/20848/ense\%C3\%B 1amos_Pozuelo_CARACCIOLOS_2014_N2.pdf?sequence=1\&isAllow ed=y

Rodríguez, B. y Luz, A. (2018). El liderazgo educativo: Una mirada etnográfica. Cuaderno de Investigación en la Educación, 26, 15-33. http://cie.uprrp.edu/cuaderno/download/numero_26/vol26_01_Benitez .pdf

Rodríguez, E. y Pedraja, L. (2017). Relación entre el liderazgo transformacional y el clima orientado al servicio de los estudiantes:

Evidencia exploratoria desde Chile. Interciencia, 42(10), 633-640.

Recuperado de: https://www.interciencia.net/wpcontent/uploads/2017/10/633-RODRIGUEZ-42_108.pdf

Rodríguez-García, A.M., Cáceres, M.P. y Alonso, S. (2018). La competencia digital del futuro docente: análisis bibliométrico de la productividad científica indexada en Scopus. International Journal of Educational Research and Innovation (IJERI), 10, 317-333. Recuperado de: https://www.upo.es/revistas/index.php/IJERI/article/download/2960/2 $737 /$

Rodríguez-García, A.M., Martínez, N. y Raso, F. (2017). La formación del profesorado en competencia digital: Clave para la educación del siglo XXI. Revista Internacional de Didáctica y Organización Educativa, 3(2), 46-65. Recuperado de: http://www.re-

doe.com/index.php?journal=reidoe $\&$ page $=$ article $\&$ op $=$ view $\&$ path $\% 5$ $\mathrm{B} \% 5 \mathrm{D}=88$

Rotellar, C. y Cain, J. (2016). Research, perspectives, and recommendations on implementing the flipped classroom. American journal of 
pharmaceutical education, 80(2), 34. Recuperado de: https://www.ncbi.nlm.nih.gov/pmc/articles/PMC4827585/

Santiago, R., Navaridas, F. y Andía, L.A. (2016). Las percepciones de los directivos de centros escolares sobre el uso y el valor de las TIC para el cambio e innovación educativa. Estudios sobre Educación, 30, 145-174. doi: 10.15581/004.30.145-174

Sola, T., Aznar, I., Romero, J.M. y Rodríguez-García, A.M. (2019). Eficacia del Método Flipped Classroom en la Universidad: Meta-Análisis de la Producción Científica de Impacto. REICE. Revista Iberoamericana sobre Calidad, Eficacia y Cambio en Educación, 17(1), 25-38.

Sosa, M.J. y Valverde, J. (2015). El Equipo Directivo «E-Competente» y su Liderazgo en el Proceso de Integración de las TIC en los Centros Educativos. Revista Iberoamericana de Evaluación Educativa, 8(2), 77103. Recuperado de:

https://dialnet.unirioja.es/descarga/articulo/5308049.pdf

Spillane, J., Camburn, E.M. y Pareja, A. (2009). School principals at work: a distributed perspective. En K. Leithwood, B. Mascall y T. Strauss (eds.), Distributed leadership according to the evidence (pp. 105-128). London: Routledge.

Suárez, J.M., Almerich, G., Díaz, I. y Fernández, R. (2012). Competencias del profesorado en las TIC. Influencia de factores personales y contextuales. Universitas Psychologica, 9(1), 293-309.

Tourón, J., Martín, D., Navarro, E., Pradas, S. y Íñigo, V. (2018). Validación de constructo de un instrumento para medir la competencia digital docente de los profesores (CDD). Revista Española de Pedagogía, 76 (269), 2554. doi: 10.22550/REP76-1-2018-02

Vacek, P. y Rybenska, K. (2015). Research of interest in ICT education among seniors. Procedia-Social and Behavioral Sciences, 171, 1038-1045. doi: 10.1016/j.sbspro.2015.01.276

Vaillant, D., Rodríguez, E. y Bernasconi, G. (2017). Modalidad MOOC para educación media básica: enseñanzas de una experiencia. Perfiles educativos, 39(156), 103-118. Recuperado de: http://www.redalyc.org/pdf/132/13250923007.pdf

Valdés, V. y Gutiérrez, P. (2018). Las Urgencias Pedagógicas en la sociedad del aprendizaje y el conocimiento. Un estudio para la reflexión sobre la 
REMIE - Multidisciplinary Journal of Educational Research, 9(2) 223

calidad en el nuevo modelo educativo. Multidisciplinary Journal of Educational Research, (8), 1-28. doi: 10.17583/remie.2018.3199

Viñals, A. y Cuenca, J. (2016). El rol del docente en la era digital. Revista interuniversitaria de formación del profesorado, 86(30), 103-114. Recuperado: https://www.redalyc.org/pdf/274/27447325008.pdf

Jesús López Belmonte, es profesor en el Departamento de Educación de la Universidad Internacional de Valencia y miembro del Grupo de Investigación AREA (Análisis de la Realidad Educativa)

Santiago Pozo Sánchez, es profesor de Didáctica y Organización Escolar de la Universidad de la Granada y miembro del Grupo de Investigación AREA

Arturo Fuentes Cabrera, es profesor en el Departamento de Métodos de Investigación y Diagnóstico en Educación de la Universidad de Granada y miembro del Grupo de Investigación AREA

José María Romero Rodríguez, es doctorando de Didáctica y Organización Escolar de la Universidad de la Granada y miembro del Grupo de Investigación AREA

Contact Address: callle Pintor Sorolla, 21. Valencia Email: jesus.lopezb@campusviu.es 